\title{
Finite Size Correction to The Thermo-Magnetic Properties of Charged Bose Gas
}

\author{
A. Hussein*, W. Seif*, Th. M. El-Sherbini* and Ahmed S. Hassan** \\ *Physics Department, Faculty of Science, Cairo University, Egypt and **Physics \\ Department, Faculty of Science, Menia University, Egypt.
}

\begin{abstract}
We consider the thermo-magnetic properties of charged boson gas confined in a quasitwo dimensional harmonic superimposed by external magnetic field in the z-direction. A modified semi-classical approximation, which is the density of state approach, is used to calculate the magnetization and magnetic susceptibility for this system. Our approach enabled us to consider the finite size correction. We get remarkable magnetic properties. The system shows diamagnetic behavior. The finite size effect decreases the transition temperature.
\end{abstract}

\section{Introduction}

At very low temperatures, the ideal charged Bose gas (CBG) in an external magnetic field became important scince Schafroth [1] showed that such a gas could exhibit a Meissner effect (this effect is the expulsion of a magnetic field from a superconductor during its transition to the superconducting state). For the ideal case, Schafroth pointed that, in the presence of a magnetic field, Bose-Einstein condensation (BEC) no longer occurs in a strict sense but gradually occurs (diffuse). Diffuse phase transitions are those not having a definite critical temperature, but an interval of temperatures along which the transition occurs gradually. The concept of diffuse was already introduced in the study of phase transitions which occur in certain ferroelectric materials [2]. A step further, Toms [3] proved that BEC in the presence of a uniform magnetic field does not occur in any number of spatial dimensions, Toms used a different definition of BEC in his approach. In this spirit, Rojas [4] found that BEC may occur in the presence of a homogeneous magnetic field, but there is no critical temperature at which condensation starts, the phase transition to BEC being diffuse. However, BEC of a $\mathrm{CBG}$ in the presence of a magnetic field in $3 \mathrm{D}$ continued to be studied by Toms and coworkers [5], who showed that, although there is no BEC in the sense of a sharp phase transition, the specific heat exhibits a clear maximum that can be used to define a critical temperature. The authors inferred that the critical temperature increases with the magnetic field, reaching the usual value for the $3 \mathrm{D}$ free Bose gas when magnetic field is absent.

However, the order parameter to investigate the magnetic properties of the system is the magnetization $M$. In general for boson system, magnetization increases continuously from zero as $\mathrm{T}$ drops below critical temperature $\mathrm{T}_{\mathrm{C}}$. The system exhibits diamagnetic behavior when the magnetization is negative $(M<0)$ and it tends to be paramagnetic if the magnetization is positive $(M>0)$ or $(M=0)$ if the magnetic moments are not pointing in any particular direction but can be aligned by the external field. If the magnetic moments are lined up and are pointing in the same direction even in the absence of the external magnetic field, then the system is ferromagnetic with a net magnetization $M \neq 0$. At high temperatures the system can be in the paramagnetic state and can undergo a phase transition into a ferromagnetic state at some temperature $T_{C}$. One signature of the phase transition is the magnetic susceptibility $\chi(\mathrm{T})$ which diverges at $\mathrm{T}=\mathrm{T}_{\mathrm{C}}$. The magnetic susceptibility tells us how easy it is for the spins to respond to a magnetic field. $\chi(\mathrm{T})$ diverges as one approaches $\mathrm{T}_{\mathrm{C}}$ from high or low temperatures.

In this paper, we focus on the magnetic properties of charged spinless Bose gases (CBG) in a quasi two dimensional harmonic potential in the presence of an external magnetic field. Our system is confined in $x y$ plane and superimposed 
with magnetic field pointed along the $z$ direction. The temperature dependence of the magnetization and magnetic susceptibility are derived taking into account the finite size effect. The calculated results show that the finite size changed the lowtemperature behavior of the magnetic properties of this system.

The outline of this paper is as follows: in the next section, the physical model of the system is written down and the modified DOS is calculated. The condensate fraction and the transition temperature are given in section 2 . The mgnetization and magnetic susceptibility are given in section 3. Finally, we summarized the conclusions in the last section.

\section{The physical model}

Let us consider a system composed of Bose gas with mass $m$ and charge $q$ which is confined in two dimensional (2D) harmonic potential. The system is exposed to a uniform magnetic field aligned along the $z$-axis, $B \wedge=B e^{\wedge} z$. The Hamiltonian can be expressed as [6]

$$
H=H_{r}+H_{z}
$$

where

$$
\begin{gathered}
H_{r}=\frac{1}{2 m}(p-A q)^{2}+\frac{1}{2} m \omega_{\perp}\left(x^{2}+y^{2}\right) \\
H_{Z}=\frac{p_{l}^{2}}{2 m}
\end{gathered}
$$

where $\omega_{\perp}=\omega_{x, y}$ is the effective trapping frequency and the gauge potential is given by:

$$
A=\frac{1}{2} B x \boldsymbol{r}
$$

The z- component of the Hamiltonian $H_{Z}$ $H_{Z}$ is associated with the translational degree of freedom of the system in the $z$-direction, with effective mass $m$. Here, the Hamiltonian

$$
H_{\varphi}=\frac{p_{x}^{2}+p_{y}^{2}}{2 m}+\frac{1}{2} m\left[(\omega]_{1}^{2}+\Omega^{2}\right)\left(x^{2}+y^{2}\right)-\Omega L_{z}
$$

Describe the behavior in xy plane. It shows that the system is trapped with frequency $\llbracket\left(\omega \rrbracket_{\downarrow} \perp^{\uparrow} 2+\Omega^{\uparrow} 2\right)$ with $q B=\frac{\Omega}{2 m}$ is the cyclotron frequency. The eigen values of the Hamiltonian $\mathrm{H}$ is given by
$E_{n_{+}, n_{-} k_{2}}=\frac{\hbar^{2} k_{l}^{2}}{2 m}+n_{+} h \omega_{+}+n_{-} h \omega_{-}+E_{0}$
Where $\left.\omega_{ \pm}=\omega \mp \Omega, E_{0}=\hbar \llbracket(\omega)_{\perp}^{2}+\Omega^{2}\right)^{\frac{1}{2}}$

and $n_{ \pm}$is non-negative integers. Clearly, the ground state for charged Bose gas has a magnetic property. the energy eigenvalues show that the system has translational degree of freedom in direction of the applied field and hyperfine vibrational degrees of freedom labelled by $n+$ in addition to degenerate Landau level indexed by $n^{-}$.

\section{Accurate DOS}

The accurate density of states for this system can be calculated using the method used by Kirsten and Toms [7], in which an asymptotic high- temperature expansion of the partition function $Z(\beta)$ is obtained. Generally, consider the eigenvalues $E k_{\mathrm{z}}, n^{+}, n^{-}$given in Eq. (4), we have,

$$
\rho_{S}(E)=\frac{4}{c_{1}} E^{\frac{3}{2}}+c_{2} E^{\frac{1}{2}}
$$

Where

$$
C_{1}=\left(\frac{m}{2 \pi^{2} \hbar^{2}}\right)^{\frac{1}{2}} \frac{1}{\hbar \omega_{+} \omega_{-}}
$$

And

$$
C_{2}=C_{1} h\left(\omega_{+}+\omega_{-}\right)
$$

The q-potential for Bose gas using the eigenvalues (4) given by,

$$
q=-\sum_{n_{+}, n_{-}, k_{z}} \ln \left(1-e^{E_{n_{+}, n_{-}, k_{z}}}\right)
$$

Expanding the logarithm to express the $\mathrm{q}$ potential as a sum over Bose-Einstein distribution and using the semiclassical approximation in which the summation over $\mathrm{n}$ is converted into an integral weighted by an the DOS [8] we have

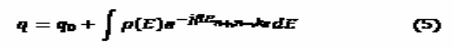

where $q o=-\ln (1-z)$ is the contribution of the ground states, $z=e^{-\beta(E 0-\mu)}$ is the effective fugacity and $\beta=1 / k T$. Substituting by $\rho_{S}(E)$, the thermodynamic potential (4) reads 


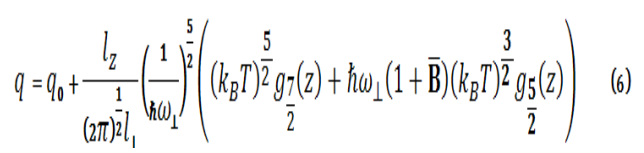

where $g_{v}(z)=\sum \frac{z^{j}}{j^{v}}$ is the usual Bose function, reduces to Riemann zeta function $\zeta(n)$ at $z=1$, $\overline{\mathbf{B}}=\frac{\Omega}{\omega_{\perp} \text { and }} l_{\perp}=\left(\frac{m}{\hbar \omega_{\perp}}\right)^{\frac{1}{2}}$

\section{Condensation fraction and critical temperature}

In terms of the q-potential, the total number of particles is $\mathrm{d}$ as

$$
N=Z \frac{\partial q}{\partial 7}
$$

Substituting from Eq.(6) we have,

$$
N=N_{0}+\frac{l_{2}}{\frac{1}{(2 \pi) l_{1}^{2}}}\left(\frac{1}{h \omega_{1}}\right)^{\frac{5}{2}}\left(\frac{5}{\left(k_{B} T\right)^{\frac{5}{2}}} g_{5}(z)+\hbar \omega_{\perp}\left(1+\bar{B}^{2}\right)\left(k_{B} B\right)^{\frac{3}{2}} g_{\frac{3}{2}}(z)\right)
$$

Where $N_{0}$ is the number of particle condensate in the ground state of the system

$$
\begin{aligned}
& \text { In terms of a reduced temperature }=\frac{T}{=} \\
&=\frac{T}{T_{0}} \text {, the condensate fraction is given by } \\
& \frac{N_{0}}{N}=1-\tau^{\frac{5}{2}}+R(\overline{\mathbf{B}}) \tau^{\frac{3}{2}}
\end{aligned}
$$

Where $\mathrm{T}_{0}$ is the BEC transition temperature for the ideal gas in the the thermodynamic limit

$$
k_{B} T_{0}=\hbar \omega_{\perp}\left(\frac{N}{\zeta\left(\frac{5}{2}\right)}\right)^{\frac{2}{5}}\left(\frac{(2 \pi)^{\frac{1}{2} l_{\perp}}}{l_{z}}\right)^{\frac{2}{5}}
$$

and

$$
R(\mathbf{B})=\left(1+\overline{\mathbf{B}}^{2}\right)\left(\frac{\zeta\left(\frac{5}{2}\right)}{N}\right)^{\frac{2}{5}}\left(\frac{l_{z}}{(2 \pi)^{\frac{1}{2}} l_{\perp}}\right)^{\frac{2}{5}} \frac{\zeta\left(\frac{3}{2}\right)}{\zeta\left(\frac{5}{2}\right)}
$$

The parameter $R(\mathbf{B})$ amounts to the finite size effect. Results in (6) shows that the condensation fraction suppressed as the magnetic field is strengthened. In Fig. 1, we illustrated this behavior graphically for different magnetic field strength. In the thermodynamic limit $N \rightarrow \infty$ the parameter $R(\mathbf{B})$ vanished and the results of ideal gas were recovered. It is important to consider the effect of the finite size on the transition temperature, which can be calculated by setting $N / N o$ in Eq. (8) equal to zero, thus

$$
T_{c}(\overline{\mathbf{B}})=T_{\mathbf{0}}\left(\mathbf{1}-\frac{\mathbf{2}}{\mathbf{5}} R(\overline{\mathbf{B}})\right)
$$

Result in (10) show that the system condenses at temperature relevant to the applied field. The transition temperature decreases as the magnetic field is strengthened which agree with the established study of superconductivity. In Fig. 2 we illustrate the effect of the magnetic field on the shift of the transition temperature. This figure shows that in thermodynamic limit and absence of the magnetic field the critical temperature is the same as the ideal gas transition temperature To. While for finite number of particle the shift increases due to the effect of finite size of particle.

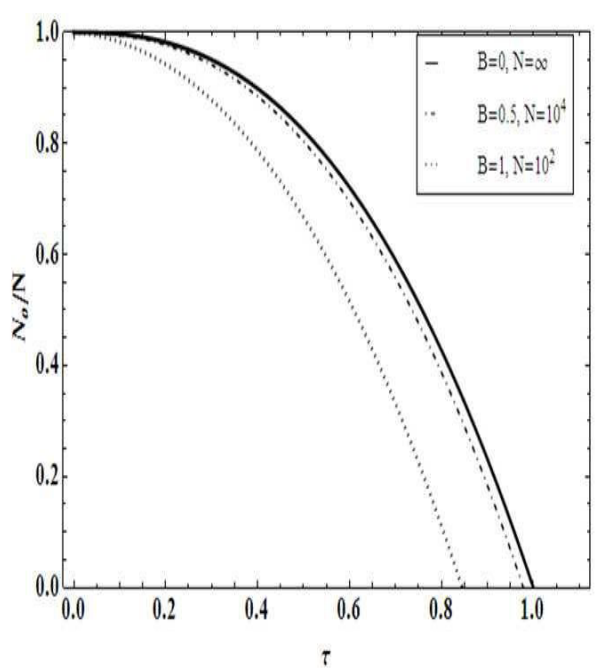

Fig. 1. Condensation fraction versus reduced temperature 


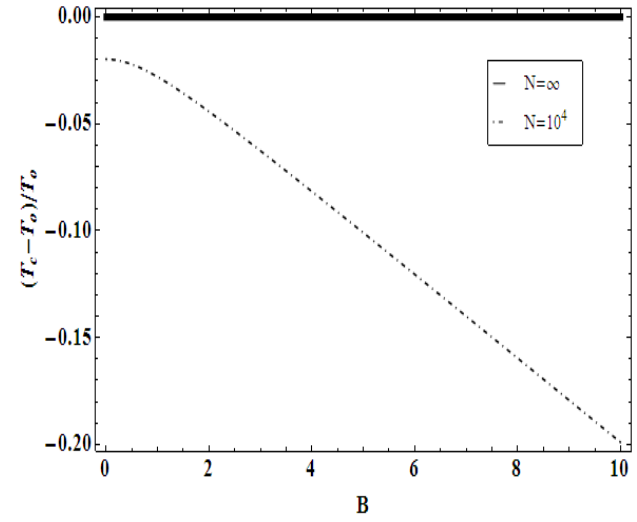

Fig. 2. Shift in the transition temperature versus the applied magnetic field.

Magnetization and magnetic susceptibility

In the semi-classical approximation the magnetization is calculated using the relation:

$$
M=-\frac{\partial q}{\partial \mathbf{B}}
$$

which yields two different behaviors for the magnetization temperature dependence. For $T>$ $T_{0}$ the condensate part $N_{0}$ vanished and and the chemical potential $\mu$ depended on the temperature. However the magnetization at temperature above $\mathrm{BEC}$ transition temperature is given by,

$M=\llbracket-\left(M I_{0}-\frac{l_{Z}}{(2 \pi)^{\frac{1}{2}} l_{\perp}}\left(\frac{1}{\hbar \omega_{\perp}}\right)^{\frac{5}{2}} \frac{\hbar \omega_{\perp} \underline{B}}{\left(1+B^{2}\right)^{\frac{1}{2}}}\left(k_{B} T\right)^{\frac{5}{2}} g_{\frac{5}{2}}(z)\right)$

Where $M_{0}$ is the magnetization due to condensate part. The total magnetization per particle in terms of the reduced temperature reads:

$$
M=-\left(M_{0}-\frac{\hbar \omega_{\perp} \underline{B}}{\left(1+\mathrm{B}^{2}\right)^{\frac{1}{2}}} \tau^{\frac{5}{2}} g_{\frac{5}{2}}(Z) / \zeta(5 / 2)\right)
$$

Thus the magnetization vanished with temperature and the magnetic field penetrated the system.

For $T \leq T o$, the chemical potential is fixed and the number of atoms in the ground state $N_{0}$ depended on the temperature, in this case the magnetization is given by

$$
M=\llbracket-\left(M \rrbracket_{0}-\frac{\hbar \omega_{\perp} \mathrm{B}}{\left(1+\mathrm{B}^{2}\right)^{\frac{1}{2}}} \tau^{\frac{5}{2}}\right)
$$

This result shows that the system exhibits strong landau diamagnetism due to the charge degree of freedom. This behavior is due to the induction of orbital motion in the material (Landau diamagnetism), results in orbital dipole moments oppose those of the external field according to Lenz rule. The induced field in diamagnetic material in extreme case may completely compensate the external field and the interior of the material is absolutely free of fields. This phenomenon is observed in superconductivity. The present result shows that $M$ vanishes as $B \rightarrow 0$ at all temperatures, implying that the Meissner Ochsenfeld effect might not exist in a trapped $\mathrm{CBG}$.

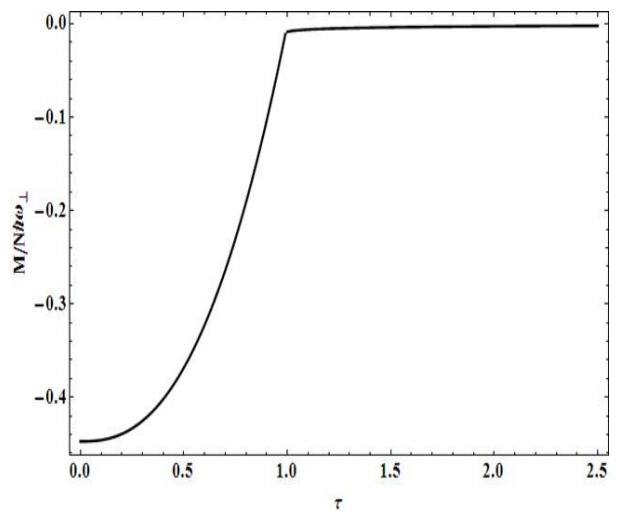

Fig. 3. Magnetization versus reduced temperature

Figure 3 illustrates the relation between the magnetization versus the reduced. This figure reveals that the system at high temperatures can be in the paramagnetic state and can then undergo a phase transition into a diamagnetism state at temperature less than the transition temperature. Above the transition temperature the system possess magnetization irrelevant to temperature but as temperature lowered below $\mathrm{T}_{0}$, the magnetization decreases monotonically to zero as $\mathrm{T}$ decreases to zero.

It's important to investigate the magnetic susceptibility to address the nature of magnetic transition. An expression for the magnetic susceptibility can be derived by inserting Eq.(12) and (13) into $\chi=\frac{\partial M}{\partial B}$ at constant temperature. However, the magnetic susceptibility above the critical temperature is given by 


$$
\chi=-\chi_{0}\left(1-\frac{g_{5}(Z)}{\zeta\left(\begin{array}{l}
5 \\
2
\end{array}\right)} \tau^{\frac{5}{2}}-\underline{B}\left(1+\underline{B^{2}}\right) \frac{g^{3}(Z)}{\zeta\left(\begin{array}{l}
\frac{2}{5} \\
2
\end{array}\right)} \tau^{\frac{3}{2}}\left(\begin{array}{l}
1 d z \\
z d B
\end{array}\right)\right.
$$

$$
\chi_{0}=\frac{\hbar \omega_{\perp}}{\left(1+\underline{B}^{2}\right)^{\frac{3}{2}}}
$$

$$
\frac{1}{Z} \frac{d z}{d \underline{B}}=-\frac{g_{\frac{5}{2}}(z)+\underline{B}\left(1+\underline{B}^{2}\right)^{\frac{1}{2}} \frac{g_{3}(z)}{\tau}}{g_{\frac{3}{2}}(z)+\underline{B}\left(1+\underline{B}^{2}\right)^{\frac{1}{2}} \frac{g_{1}(z)}{\tau}}
$$

whereas below the critical temperature is given by:

$$
\chi=-\chi_{0}\left(1-\tau^{\frac{5}{2}}\right)
$$

Results in Eq. (14) and (16) show that the system exhibits a strong diamagnetism below the transition temperature. This behavior can be seen clearly by representing the results graphically.

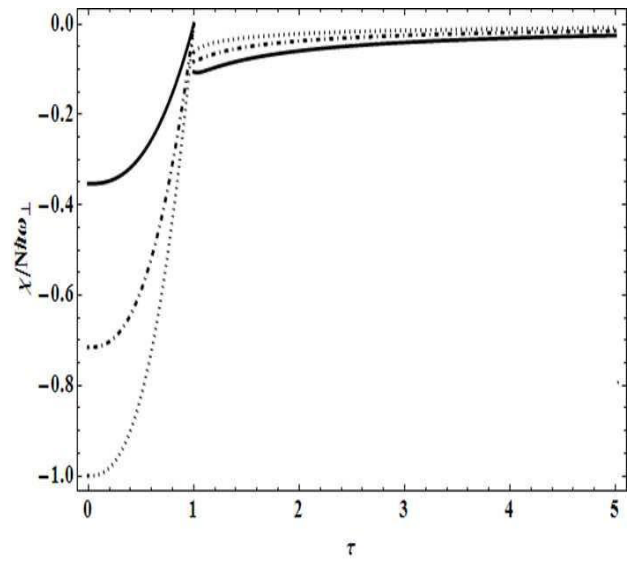

Fig. 4. Magnetic susceptibility versus reduced temperature for different magnetic field strength.

In Fig. 4, the magnetic susceptibility versus reduced temperature for different magnetic field strength is given. This figure shows that the magnetic susceptibility has a negative value approximately constant at very low temperature. The temperature dependence of the magnetic susceptibility appeared as temperature rose; it reached zero at $T_{0}$ at which the magnetic field penetrated the system. For sufficient strong value of the external magnetic field, the system displays a diamagnetic behavior above $T_{0}$ with negative susceptibility. Again the susceptibility goes to zero at relatively high temperature.

\section{Conclusion}

In this paper the semiclassical approximation is used to calculate the critical temperature, the magnetization and the magnetic susceptibility for a system of charged boson gas confined in quasi-two dimensional harmonic potential in the presence of an external magnetic field pointed in the z-direction. The calculated results are modified significantly due to inclusion of the finite size. The transition temperature decreases with the increases of the external magnetic field. The magnetization vanishes when the magnetic field is reduced to zero, implying that the MeissnerOchsenfeld effect might not exist. Moreover, the magnetic susceptibility is negative at temperature less than the transition temperature, consequently the system exhibits diamagnetic properties.

\section{References}

1. Schafroth, M. R. Phys. Rev. 100, 476 (1955).

2. Smolenski,G.A. and Isupov,V. A., Sov. Journal of Techn. Phys. 24, 1375 (1954).

3. Toms, D. J. , Phys. Rev. Lett. 69, 1152 (1992).

4. Rojas,H. P. , Phys. Lett. B 379, 148 (1996)., H. P. Rojas, Phys. Lett. A 234, 13 (1997).

5. Standen,G.B. and Toms,D. J., Phys. Lett. A 239, 401 (1998)., G. B. Standen and D. J. Toms, Phys. Rev. E 60, 5275 (1999).

6. Fetter,A. L., Rev. Mod. Phys. 81, 647 (2009).

7. Kirsten,K. and Toms,D. J. , Phys. Lett. A222 (1996) 148; Phys. Rev. A54 (1996) 4188;Kirsten, K. and Toms,D.J. Phys. Rev. E59 (1999) 158; Kirsten, K. and Toms,D. J., Phys. Lett. A243, 137 (1998) .

8. Hassan,A. S., Phys. Lett. A 374 (2010) 2106; Hassan, A. S. , El-Badry, A. M. and Soliman, S. S. M., Eur. Phys. J. D. 64 (2011) 465; Hassan, A. S., El-Badry,A. M. , Mohammedein, A. M., Ebeid, M.R., Phys. Lett. A 376, 1781 (2012).

(Received:17/7/2017; accepted:20/8/2017) 
Finite size correction to the thermomagnetic properties of charged Bose gas

عباس حسين* ، و لاء محمد سيف* ، ثروت الثربينى * و احمد سيد حسن**

قسم الفيزياء-كلية العلوم- جامعة القاهرة*

(*قسم الفيزياء-كلية العلوم- جامعة المنيا

فى هذه البحث تم در اسة الخو اص الثيرمومغناطيسية لجسيمات بوز المشحونة فى مصيدة جهد

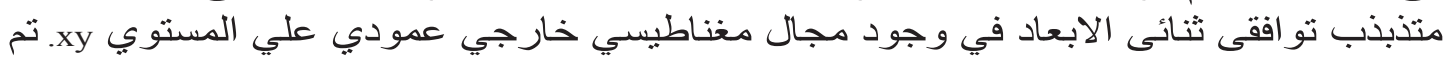
استخدام التقريب شبه الكلاسيكى المعدل الذي يمكن الذي من اخذ تصحيح محدودية الحجم في الاعتبار .

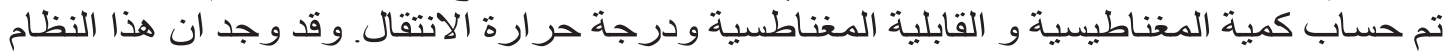

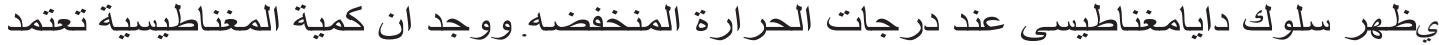
على درجة الحرارة وكذلك شدة المجال المغناطيسى. 